University of Nebraska - Lincoln

DigitalCommons@University of Nebraska - Lincoln

\title{
Seeding Effect on Micro- and Domain Structure of Sol-Gel- Derived PZT Thin Films
}

A. L. Kholkin

University of Aveiro, 3810-193 Aveiro, Portugal, kholkin@cv.ua.pt

Alexei Gruverman

University of Nebraska-Lincoln, agruverman2@unl.edu

A. Wu

University of Aveiro, 3810-193 Aveiro, Portugal

M. Avdeev

University of Aveiro, 3810-193 Aveiro, Portugal

P. M. Vilarinho

University of Aveiro, 3810-193 Aveiro, Portugal

See next page for additional authors

Follow this and additional works at: https://digitalcommons.unl.edu/physicsgruverman

Part of the Physics Commons

Kholkin, A. L.; Gruverman, Alexei; Wu, A.; Avdeev, M.; Vilarinho, P. M.; Salvado, I. M. Miranda; and Baptista, J. L., "Seeding Effect on Micro- and Domain Structure of Sol-Gel-Derived PZT Thin Films" (2001). Alexei Gruverman Publications. 48.

https://digitalcommons.unl.edu/physicsgruverman/48

This Article is brought to you for free and open access by the Research Papers in Physics and Astronomy at DigitalCommons@University of Nebraska - Lincoln. It has been accepted for inclusion in Alexei Gruverman Publications by an authorized administrator of DigitalCommons@University of Nebraska - Lincoln. 


\section{Authors}

A. L. Kholkin, Alexei Gruverman, A. Wu, M. Avdeev, P. M. Vilarinho, I. M. Miranda Salvado, and J. L. Baptista 
Published in Materials Letters 50:4 (September 2001), pp. 219-224; doi 10.1016/S0167-577X(01)00228-2

Copyright ( 2001 Elsevier Science B.V. Used by permission.

http://www.sciencedirect.com/science/journal/0167577X

Submitted November 15, 2000; accepted December 5, 2000; published online September 4, 2001.

\title{
Seeding effect on micro- and domain structure of sol-gel-derived PZT thin films
}

\author{
A. L. Kholkin, ${ }^{1}$ A. Gruverman, ${ }^{2}$ A. Wu, ${ }^{1}$ M. Avdeev, ${ }^{1}$ P. M. Vilarinho, ${ }^{1}$ \\ I. M. Miranda Salvado, ${ }^{1}$ and J. L. Baptista ${ }^{1}$ \\ ${ }^{1}$ Department of Ceramics and Glass Engineering, University of Aveiro, 3810-193 Aveiro, Portugal \\ ${ }^{2}$ Department of Materials Science and Engineering, North Carolina State University, \\ Raleigh, NC 27695-7919, USA \\ Corresponding author - A. L. Kholkin; email kholkin@cv.ua.pt
}

\begin{abstract}
Current trend to miniaturization requires precise control of micro- and domain structure of ferroelectric materials at the nanoscale level. This is essential for future applications of ferroelectric thin films in non-volatile memories, microactuators and pyroelectric arrays. In this work, the seeding effect on the nanoscale properties of ferroelectric $\mathrm{Pb}(\mathrm{Zr}, \mathrm{Ti}) \mathrm{O}_{3}(\mathrm{PZT})$ thin films is investigated using scanning force microscopy (SFM) capable of simultaneously resolving topographic and domain features on the surface of the films. It is shown that the addition of $5 \mathrm{~mol} \%$ seeds (fine PZT powder) into the sol-gel precursor solution completely modifies the film's microstructure leading to the improved morphology of the grains, reduced roughness and smaller microporosity. At the same time, significant imprint and instability of the written domain pattern is reduced due to the smaller influence of the bottom electrode interface.
\end{abstract}

Keywords: SFM, sol-gel, $\mathrm{PZT}\left(\mathrm{Pb}(\mathrm{Zr}, \mathrm{Ti}) \mathrm{O}_{3}\right)$ films, ferroelectric domains

\section{Introduction}

Ferroelectric thin films such as lead zirconate titanate (PZT) are of great interest for various applications including non-volatile memories, piezoelectric microactuators and pyroelectric detectors [1]. Current trend to miniaturization requires scaling down of the lateral dimensions of the devices to few micrometers where the scale of the microstructural and domain features becomes comparable to the size of the active part of the film. This will inevitably lead to a large statis- tical variation in the performance of the devices which may consist of just few grains or domains. Therefore, more uniform and fine micro- and domain structure of ceramic films is required for microelectronic applications. Another problem is the relatively high processing temperatures of $\mathrm{Pb}$-based films, which limits their direct integration into the high-density Si circuitry [2]. A novel approach to both improve the microstructure and to decrease the processing temperatures has been recently demonstrated by $\mathrm{Wu}$ et al. [3-5], who introduced a small amount of fine ferroelectric 
powders into the sol-gel PZT precursor solution used for thin film deposition. The powder particles dispersed in the solution served as the additional nucleation sites and significantly eased the crystallization of PZT thin films. Thus, lower crystallization temperatures and faster transformation of intermediate pyrochlore to the required perovskite phase could be achieved $[3,4]$. In this work, scanning force microscopy (SFM) capable of simultaneous topographic and domain imaging is used to study seeding effect on the microand domain structure of PZT films grown on Ptcoated Si substrates.

\section{Experimental procedure}

Domain imaging was provided by a commercial SFM setup (Seiko SPI 3700) equipped with a goldcoated $\mathrm{Si}_{3} \mathrm{~N}_{4}$ cantilever having a spring constant of $0.1 \mathrm{~N} / \mathrm{m}$ and a resonant frequency of $34 \mathrm{kHz}$. The essence of piezoelectric domain imaging technique is described in detail elsewhere [6 and 7] and can be briefly introduced as follows. During standard topography imaging in a contact mode, the small ac voltage $(\approx 3 \mathrm{~V}$ peak-to-peak) is applied between the conductive tip and bottom electrode. The output from SFM photodetector is fed to a lock-in amplifier and imaged along with the common Z-control feedback signal. The contrast is due to the piezoelectric effect in every individual domain because the part of the film in contact with the conductive tip deforms in accordance with the value and sign of the local piezoelectric coefficient $\left(d_{33}\right)$ of the film. Both the polarity and the absolute value of $d_{33}$ (which is proportional to local polarization) could be imaged with the nanoscale resolution. Using the SFM capabilities, the film can be locally poled, i.e., its domain structure can be intentionally modified in some locations to store information permanently as a stable domain pattern. The small thickness of ferroelectric domain walls allows for a very dense memory to be achieved (see [8]).

PZT films of the composition $\mathrm{PbZr}_{0.52} \mathrm{Ti}_{0.48} \mathrm{O}_{3}$ were prepared by sol-gel technique using lead acetate, zirconium acetylacetonate and titanium diisopropoxide bisacetylacetonate as starting chemicals. The detailed procedure for preparing the precursor solution has been reported elsewhere [3]. Fine PZT powders with the same com- position (Zr/Ti ratio 52:48) and small particle size ( 20-80 nm) were also prepared by sol-gel technique [4]. The seeds were dispersed in a solution of 1,2-propanediol, acetone and dispersant (ammonium salt of polycarboxylic acid) and the suspension was then mixed with the PZT stock solution according to the concentration of seeds 5 $\mathrm{mol} \%$. Thin PZT films were dip-coated onto Pt/ $\mathrm{Ti} / \mathrm{SiO}_{2} / \mathrm{Si}$ substrates using a withdrawal speed of $\approx 1.7 \mathrm{~mm} / \mathrm{s}$. The films were then dried at $300^{\circ} \mathrm{C}$ on a hot plate to remove residual organics. This procedure was repeated four times to achieve the final thickness of the films of about $300 \mathrm{~nm}$. Finally, the films were fired at $650^{\circ} \mathrm{C}$ for $15 \mathrm{~min}$. Pure perovskite phase was observed in both unseeded and seeded films as confirmed by XRD analysis. It is essential that unseeded films were strongly (111) oriented and seeded films showed more random orientation of the grains [5].

\section{Results and discussion}

It is well known that the perovskite phase formation of lead-based ferroelectric films prepared by wet chemical techniques is a sequential process where the desired perovskite phase is formed through intermediate pyrochlore phase. Even a small amount of pyrochlore phase has detrimental effect on the dielectric and piezoelectric properties of the films [2]. The conversion of pyrochlore into the perovskite phase is a nucleation-controlled process where the nucleation of the perovskite phase is the rate-controlling step of the phase transformation. Nucleation sites are typically provided by the underlying substrate, and columnar grains result from the crystallization front moving from the substrate to the surface of the film [2]. To a large extent, the quality of ceramic films is determined by the quality of a substrate where the lattice matching provide nucleation sites for PZT films to grow preferentially along one of the crystallographic directions. The surface morphology of unseeded PZT films is shown in Figure 1. Topography (z-deflection at constant force) is represented in Figure 1(a) while corresponding domain patterns are depicted in Figure $1(b, c)$. Large grains of irregular shape (or conglomerates of smaller grains), large microporosity and relatively rough surface of the films are clearly seen. The roughness averaged over $5 \times 5$ 
(a)

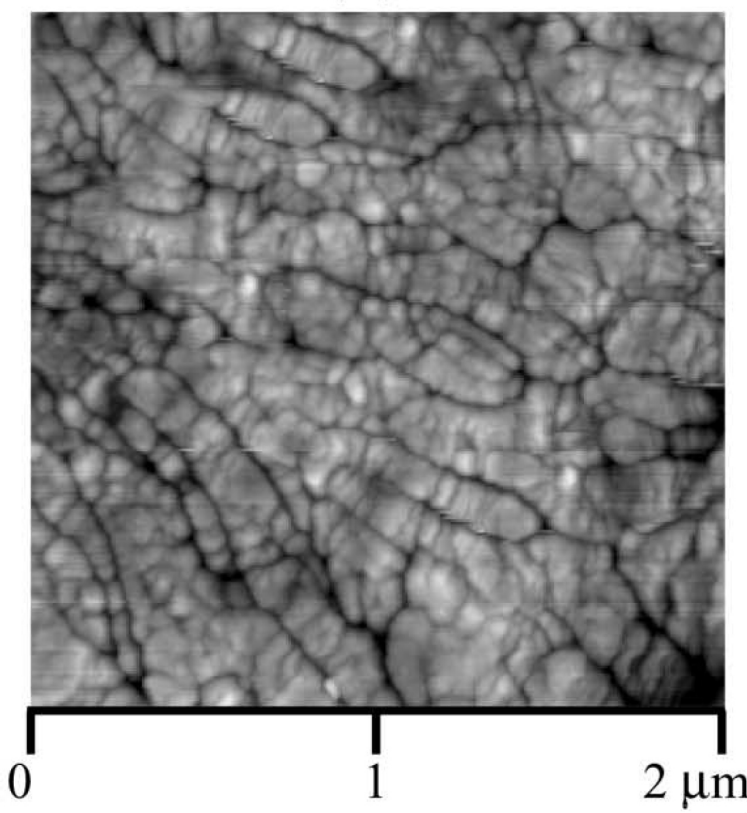

(b)

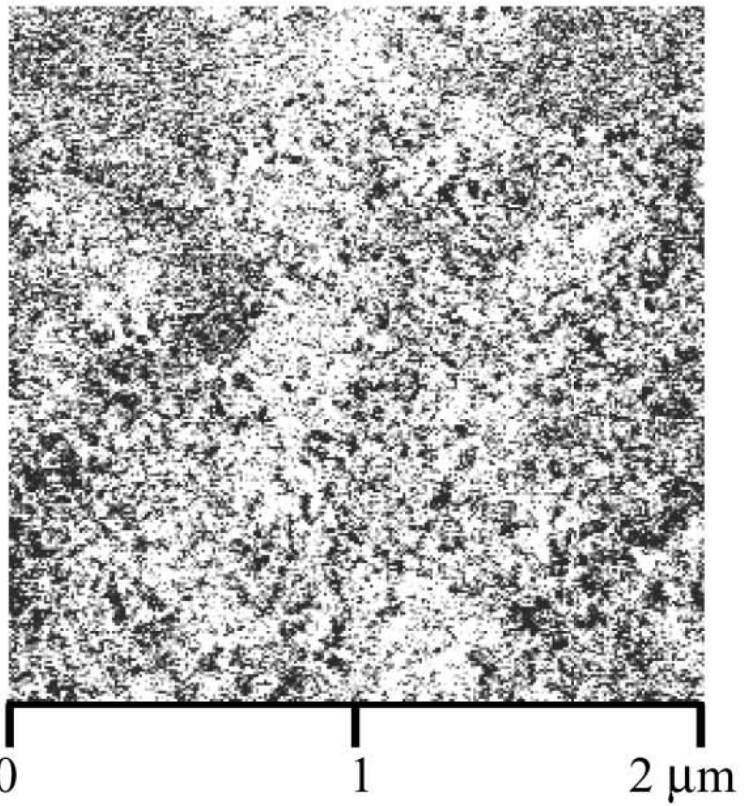

(c)



Figure 1. Topography (a) and piezoelectric (b, c) images of unseeded PZT films. "Positive" domain pattern is written in the center (c) with $V=+6 \mathrm{~V}$ applied to the tip. 
(a)

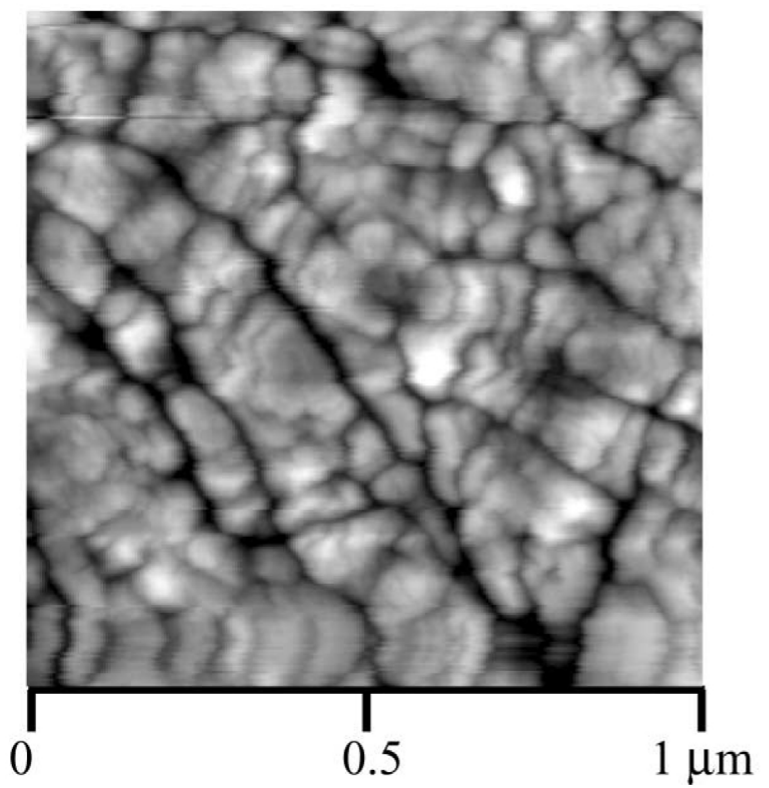

(b)

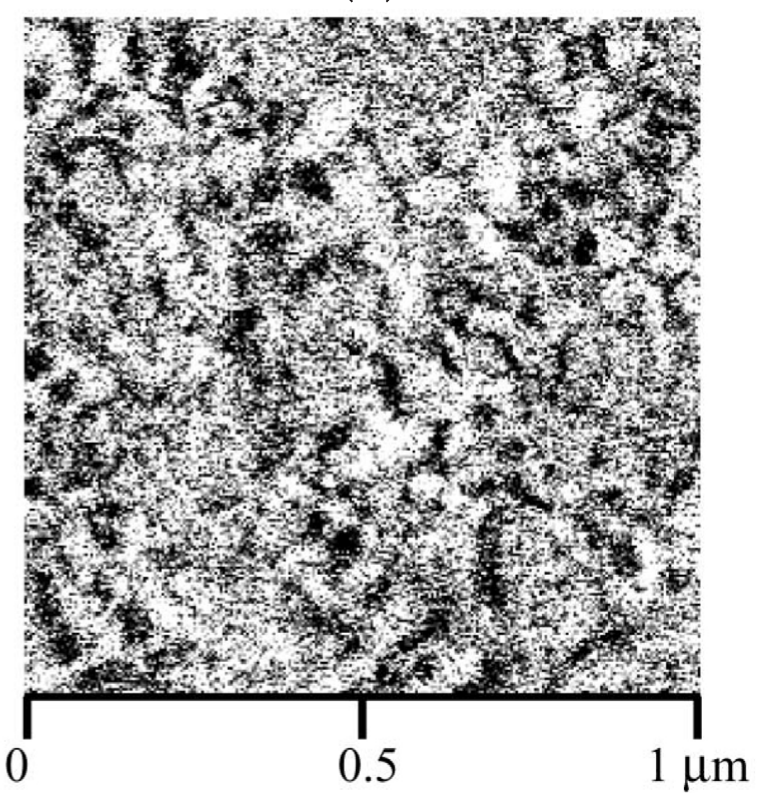

Figure 2. Topography (a) and piezoelectric (b) images of unseeded PZT films at larger magnification $(1 \times 1$ $\left.\mu \mathrm{m}^{2}\right)$.

$\mu \mathrm{m}^{2}$ area was about $66 \AA$. It has been previously suggested that the morphology and preferred orientation of the grains in PZT films grown on $\mathrm{Pt}$ electrodes are determined by the presence of $\mathrm{Pt}_{x} \mathrm{~Pb}$ intermetallic alloy, which provides better lattice matching with PZT than Pt [9]. Among other reasons, the grain structure shown in Figure 1(a) might be due to irregular defects on the surface of Pt bottom electrode, which prevent uniform nucleation of the perovskite phase. Domain structure shown in Figure 1(b) is very fine but nevertheless could be resolved when scanning at a larger magnification (Figure 2(b). Areas with different domain orientations are clearly seen and their contribution to overall piezoelectric properties of virgin films could be defined. The common feature of Figure 1 and Figure 2 is that the local piezoelectric coefficient averaged over the entire scanning area is not zero as can be expected from unpoled film with the zero net piezoelectric coefficient but has some "negative" value (in the sense defined below) corresponding to some initial polarization of the films. It has been found that at present experimental conditions, domains with the polarization vector oriented toward the bottom electrode (designated as "positive" do- mains, or "positive" polarization) appeared as dark regions, while domains oriented upward ("negative" domains) emerged as bright regions. The built-in initial polarization (self-polarization of virgin films) has been previously observed in PZT films prepared by different techniques [1012]. Among other reasons, the self-polarization effect was suggested to arise due to the internal bias field existing in the vicinity of the film/electrode interface [12]. This field could easily polarize the film during cooling down from the deposition/ crystallization temperature and cause significant imprint, i.e., preferential orientation of the polarization over the antiparallel direction. Figure 1(c) demonstrates instability of the "positive" polarization state created by the tip-induced poling of the films. The poling was done by scanning over the area of about $1 \mu \mathrm{m}^{2}$ with $V=+6 \mathrm{~V}$ applied to the tip. Few minutes after poling, the "positive" polarization directed antiparallel to initial self-polarization is almost destroyed due to significant imprint and accelerated depoling. This property is apparently detrimental for applications based on polarization switching and must be minimized by choosing appropriate deposition and/or annealing strategies [12]. It was suggested that the 


\section{(a)}

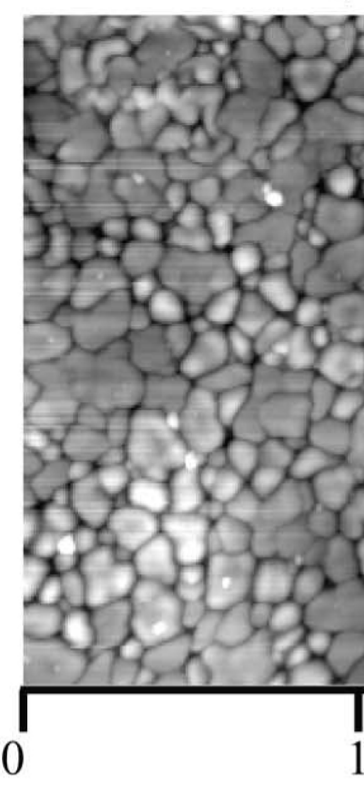

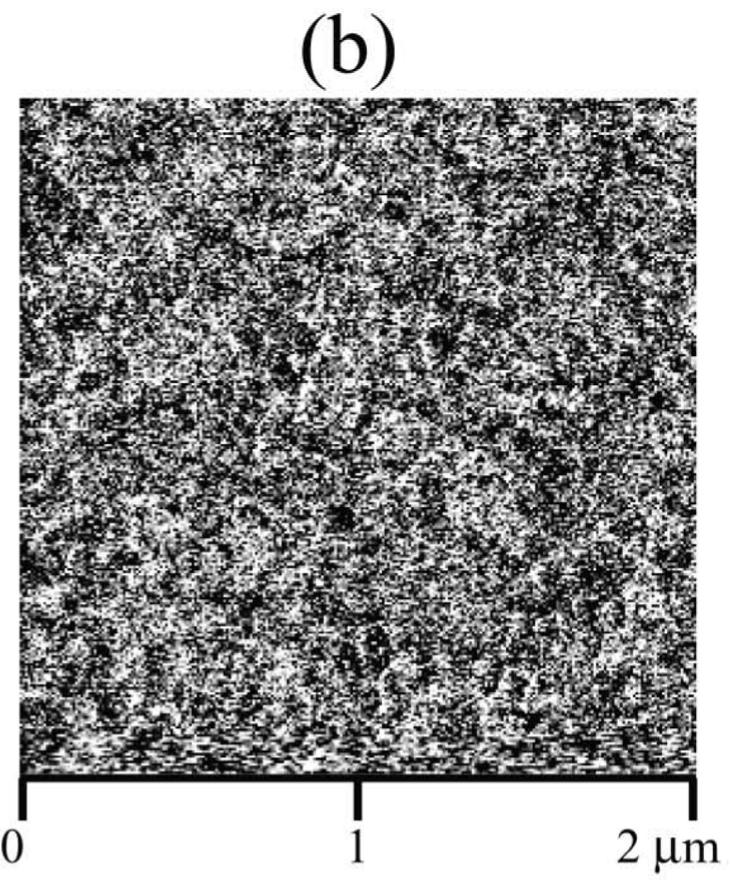

(b)

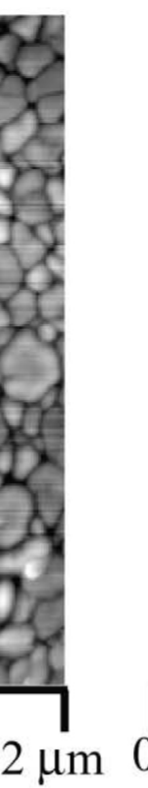

(c)

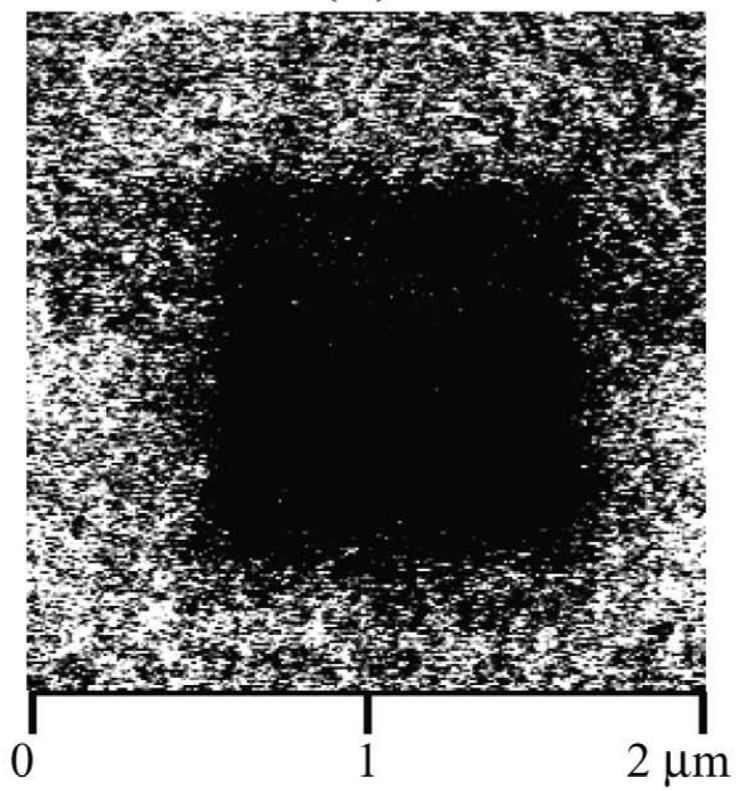

Figure 3. Topography (a) and piezoelectric (b, c) images of PZT films with $5 \mathrm{~mol} \%$ of seeds. "Positive" domain pattern is written in the center (c) with $V=+6 \mathrm{~V}$ applied to the tip (c).

internal bias field is related to Schottky barrier between the film and bottom electrode, and can be reduced by both changing the electrode and top electrode annealing [12]. Another solution would be the addition of seeds into the bulk of the films that will inevitably weaken the role of bottom 
electrode interface in the film's micro- and domain structure.

Figure 3 shows topographic and piezoelectric images of PZT film deposited with $5 \mathrm{~mol} \%$ PZT seeds. The microstructure of the films is completely modified as compared to unseeded films (Figure 1). Namely, the grain structure becomes more dense and closely packed having essentially bimodal distribution of the grains with two maxima located at around $30-40$ and $60-70$ $\mathrm{nm}$. At the same time, we observed that the averaged roughness was reduced to about $23 \AA$. It is hypothesized that the dense microstructure of seeded films is a result of the competitive influence of the nucleation controlled by both the $\mathrm{Pt}$ electrode interface and by the seeds. As a result, a significant part of the grains nucleates and grows from the bottom electrode to the surface of the films, while other grains originate from PZT seeds randomly distributed in the PZT solgel matrix. PZT seeds mixed with precursor sol may also reduce porosity of the films since the composite structure is more resistive to mechanical stress arising during drying and firing procedures. It is worth mentioning that the domain contrast shown in Figure 3(b) has an averaged $d_{33}$ close to zero. No preferred polarization state is created and written domain pattern is very stable as shown in Figure 3(c). The origin of the reduced self-polarization and built-in internal field can be due to the bulk nucleation of the grains. In this case, preferred orientation and lattice matching with the electrode interface could be partly lost, and internal field of a Schottky barrier might be smaller since it is determined by the quality of the interface. We believe that this situation is closer to the case of bulk ceramics where self-polarization and fast imprint are not typically observed even in very thin samples.

\section{Conclusions}

In conclusion, it has been observed that addition of $5 \mathrm{~mol} \%$ of fine PZT seeds has significant effect on micro- and domain structure of sol-gel-derived PZT films. The films prepared with $5 \mathrm{~mol} \%$ seeds showed denser and better-defined microstructure with reduced microporosity. In seeded films, the initial polarization value and polarization imprint are reduced due to a smaller influ- ence of the bottom electrode interface. A stable domain pattern could be written on seeded films over the area of $1 \mathrm{\mu m}^{2}$.

\section{Acknowledgments}

A. Wu gratefully acknowledges the F.C.T. for funding her scholarship (PRAXIS/XXI/BPD 20196/99).

\section{References}

1. O. Auciello, J.F. Scott and R. Ramesh, The physics of ferroelectric memories. Phys. Today (1998), pp. 22-27 July.

2. B.A. Tuttle and R.W. Schwartz, Solution deposition of ferroelectric thin films. MRS Bull. (1996), pp. 49-54 June.

3. A. Wu, I.M. Miranda Salvado, P.M. Vilarinho and J.L. Baptista, Processing and seeding effects on crystallization of PZT thin films from sol-gel method. J. Eur. Ceram. Soc. 17 (1997), pp. 1443-1452.

4. A. Wu, P.M. Vilarinho, I.M. Miranda Salvado and J.L. Baptista, Seeding studies in PZT thin films. Mater. Res. Bull. 33 (1998), pp. 59-68.

5. A.Wu, L. Yang, P.M. Vilarinho, I.M. Miranda Salvado, J.L. Baptista, Preferred orientation and electrical properties of seeded PZT thin films, Thin Solid Films, in press.

6. A. Gruverman, O. Auciello and H. Tokumoto, Scanning force microscopy for the study of domain structure in ferroelectric thin films. J. Vac. Sci. Technol., B 14 (1996), pp. 602-605.

7. A. Gruverman, O. Auciello, J. Hatano and H. Tokumoto, Scanning force microscopy as a tool for nanoscale study of ferroelectric domains. Ferroelectrics 184 (1996), pp. 11-20.

8. A. Gruverman, O. Auciello and H. Tokumoto, Imaging and control of domain structures in ferroelectric thin films via scanning force microscopy. Annu. Rev. Mater. Sci. 28 (1998), pp. 101-123.

9. S.Y. Chen and I.W. Chen, Temperature-time-texture transition of $\mathrm{Pb}(\mathrm{Zr}, \mathrm{Ti}) \mathrm{O}_{3}$ thin films. J. Am. Ceram. Soc. 77 (1994), pp. 2332-2444.

10. R. Bruchaus, D. Pitzer, R. Primig, W. Wersing and Y. $\mathrm{Xu}$, Deposition of self-polarized PZT Films by planar multi-target sputtering. Integr. Ferroelectr. 14 (1997), pp. 141-149.

11. I. Kanno, S. Fujii, T. Kamada and R. Takayama, Piezoelectric properties of $c$-axis oriented $\mathrm{Pb}(\mathrm{Zr}, \mathrm{Ti}) \mathrm{O}_{3}$ thin films. Appl. Phys. Lett. 70 (1997), pp. 1378-1380.

12. A.L. Kholkin, K.G. Brooks, D.V. Taylor, S. Hiboux and N. Setter, Self-polarization effect in $\mathrm{Pb}(\mathrm{Zr}, \mathrm{Ti}) \mathrm{O}_{3}$ thin films. Integr. Ferroelectr. 22 (1998), pp. 525-533. 